\title{
On the construction of multi-valued concurrent dynamic logics
}

\author{
Leandro Gomes \\ HASLab INESC TEC - Univ. Minho, Portugal
}

\begin{abstract}
Dynamic logic is a powerful framework for reasoning about imperative programs. An extension with a concurrent operator, called concurrent propositional dynamic logic (CPDL) [20], was introduced to formalise programs running in parallel. In a different direction, other authors proposed a systematic method for generating multi-valued propositional dynamic logics to reason about weighted programs [15]. This paper presents the first step of combining these two frameworks to introduce uncertainty in concurrent computations. In the proposed framework, a weight is assigned to each branch of the parallel execution, resulting in a (possible) asymmetric parallelism, inherent to the fuzzy programming paradigm $[23,2]$. By adopting such an approach, a family of logics is obtained, called multi-valued concurrent propositional dynamic logics $(\mathcal{G C D} \mathcal{L}(\mathbf{A}))$, parametric on an action lattice $\mathbf{A}$ specifying a notion of "weight" assigned to program execution. Additionally, the validity of some axioms of CPDL is discussed in the new family of generated logics.
\end{abstract}

\section{Introduction}

Over time, the different variants of dynamic logics developed in different verification communities went hand-in-hand with the very notion of its object, the program. This resulted in a diverse myriad of dynamic logics tailored to specific programming paradigms. Examples include probabilistic [12], concurrent [20], quantum [1] and continuous [21] computations, and combinations thereof. An example of another non-trivial paradigm is the fuzzy one $[23,2]$, where the execution of a program differs from both classical and probabilistic scenarios: a conditional statement is interpreted as a concurrent execution with a weight associated to each branch. The formalisation of this sort of behaviour entails the need to address both concurrency and uncertainty. An extensive research can be found in the literature on diverse formalisms to reason about programs running in parallel $[10,11]$ and to deal with uncertainty $[12,5,22,4]$. However, even when these two components are combined into a single framework [18], the emerging paradigm is probabilistic nondeterminism. Thus we are still missing a proper semantics to describe the behaviour of the fuzzy paradigm.

* This work was founded by the ERDF — European Regional Development Fund through the Operational Programme for Competitiveness and Internationalisation - COMPETE 2020 Programme and by National Funds through the Portuguese funding agency, FCT - Fundação para a Ciência e a Tecnologia, within project POCI-01-0145-FEDER-030947. 
Reference [15] initiated a research agenda on the systematic development of multi-valued propositional dynamic logics, parametric on an action lattice, which defines both the computational paradigm where programs live, and the truth space where assertions are evaluated. Following another research line, an extension to propositional dynamic logic (PDL) was introduced in reference [20], called concurrent propositional dynamic logic $(C P D L)$, to reason about concurrent computations. In the models of this logic, the programs are interpreted as binary multirelations, to describe a parallel execution from a state to a set of states.

Combining these two research lines, this paper takes a first step on the development of a method to generate multi-valued concurrent propositional dynamic logics. As in [15], the logics are parametric on a generic action lattice, to model both the computational domain and a (possible graded) truth space where the assertions about programs are evaluated. First, the semantics of CPDL is adapted to model programs as weighted parallel executions, by introducing the concept of fuzzy multirelations. This means that a program is interpreted as a relation between a state and a fuzzy set of states. The intuition is that the weights of the fuzzy set describe an execution probability for each branch of the program, an asymmetric parallel flow or the energy/costs associated to each branch. Then, on a second stage, a method of generating (parametric) multi-valued CPDL is presented. The family of the resulting logics is called $\mathcal{G C D} \mathcal{L}(\mathbf{A})$.

This paper is organised as follows. Section 2 presents a brief background overview. Then, Section 3 introduces fuzzy multirelations and defines some operations on them. Such an algebra is the mathematical formalism in which programs are interpreted in the generated logics. The same section ends with the study of an axiomatisation for the generated logics. Finally, Section 4 concludes and enumerates topics for future work.

\section{Preliminaries}

\subsection{Semantics for concurrency}

The semantics of CPDL is based on the concept of binary multirelation. The relevant definition and some operators are recalled below.

Definition 1 (Binary multirelation [7]). Given a set X, a binary multirelation is a subset of the cartesian product $X \times 2^{X}$, i.e. a set of ordered pairs $(a, A)$, where $a \in X$ and $A \subseteq X$. The following operations over multirelations are defined:

$-R \cup S$ as the union of $R$ and $S$;

- the Peleg sequential composition

$$
R \cdot S=\{(a, A) \mid \exists B .(a, B) \in R \wedge \exists f .(\forall b \in B .(b, f(b)) \in S) \wedge A=\bigcup f(B)\} ;
$$

- the parallel composition $R \cap S=\{(a, A \cup B) \mid(a, A) \in R \wedge(a, B) \in S\}$. 
Note that the union of binary multirelations is just the set union. The sequential composition operator is rather more complex. A pair $(a, A)$ belongs to the sequential composition of multirelations $R$ and $S$ if and only if $a$ is related with some intermediate set of states $B$ and every $b \in B$ must be related with some subset of $A$ such that the union of all those subsets is $A$. Finally, an element $(a, A) \in R \cap S$ indicates a parallel execution of a program from a state $a$ to a set of states in $A$, "combining" the arriving states of $R$ and $S$ into $A$. Note that such composition is dual to $R \cup S$, where $(a, B)$ and $(a, C)$ correspond to distinct executions. The first kind of choice in commonly called demonic, while the latter is known as angelic [17]. Angelic nondeterminism represents achoice made by an 'angel', an thus, it occurs when the best possible choice is made. Demonic nondeterminism represents a choice made by a 'demon', i.e. the worst case scenario.

\subsection{Concurrent propositional dynamic logic}

Concurrent propositional dynamic logic (CPDL), as introduced in [20], is an extension of PDL with a parallel operator $\cap$ added to the syntax of programs. The semantics interprets programs as binary multirelations $R \subseteq W \times 2^{W}$, where composed programs are interpreted according to Definition 1 . Intuitively, an element $(a, A)$ of a binary multirelation expresses that the a program executed from a state $a$ ends in all states of $A$ in parallel. Models of CPDL consist of tuples $(W, V, \llbracket-\rrbracket)$ where $W$ is a set of states, $V$ is a valuation function which attributes a subset of $W$ to each atomic formula, and $\llbracket-\rrbracket$ attributes a subset of $W \times 2^{W}$ to each atomic program. For instance, the formula $\langle\pi\rangle \rho$ holds in a state $w$ if and only if $\exists U \subseteq W$ such that $(s, U) \in \llbracket \pi \rrbracket$ and $U \in V(\rho)$. For more details about the semantics of CPDL see [20]. The axiom system of CPDL is that of PDL with the additional axiom $\left\langle\pi_{1} \cap \pi_{2}\right\rangle \rho \equiv\left\langle\pi_{1}\right\rangle \rho \wedge\left\langle\pi_{2}\right\rangle \rho$ and restricting $\left\langle\pi_{0}\right\rangle\left(\rho \vee \rho^{\prime}\right) \equiv\left\langle\pi_{0}\right\rangle \vee\left\langle\pi_{0}\right\rangle \rho^{\prime}$ to atomic programs.

\subsection{Parametric construction of multi-valued dynamic logics}

Thus subsection provides a short review of the 'dynamisation' method introduced in [15]. Let us start by revisiting the following definition:

Definition 2 ([13]). An action lattice is a tuple $\mathbf{A}=(A,+, ;, 0,1, *, \rightarrow, \cdot)$, that is a residuated lattice with order $\leq$ induced $b y+: a \leq b$ if and only if $a+b=b$, plus the axioms $1+a+\left(a^{*} ; a^{*}\right) \leq a^{*}$ and $(x \rightarrow x)^{*}=x \rightarrow x$.

An action lattice is called a II-action lattice when the identity of the ; operator coincides with the greatest element of the residuated lattice, i.e. $1=\top$. Moreover, an action lattice $\mathbf{A}$ is complete when every subset of $\mathbf{A}$ has both supremum and infimum. Since operators + and ; are associative and have identity, we can generalise them to $n$-ary operators and use the notation $\sum$ and $\prod$ to represent their iterated versions, respectively. The generation of dynamic logics illustrated in the Section 3 will be parametric on the class of complete action lattices, since 
completeness is required to ensure the existence of arbitrary suprema. The general construction of multi-valued dynamic logics is revisited bellow.

Signatures. Signatures of $\mathcal{G} \mathcal{D} \mathcal{L}(\mathbf{A})$ are pairs ( $\Pi$, Prop) corresponding to the denotations of atomic programs and propositions, respectively.

Formulæ. The set of programs, denoted by $\operatorname{Prg}(\Pi)$, contains all expressions generated by $\pi \ni \pi_{0}|\pi ; \pi| \pi+\pi \mid \pi^{*}$ for $\pi_{0} \in \Pi$. Given a signature (I, Prop), the $\mathcal{G} \mathcal{D} \mathcal{L}(\mathbf{A})$-formulæ for ( $\Pi$, Prop) are the ones generated by the grammar $\rho \ni \top|\perp| p|\rho \vee \rho| \rho \wedge \rho|\rho \rightarrow \rho| \rho \leftrightarrow \rho|\langle\pi\rangle \rho|[\pi] \rho$ for $p \in$ Prop and $\pi \in \operatorname{Prg}(\Pi)$.

Semantics. The space where the computations of $\mathcal{G} \mathcal{D} \mathcal{L}(\mathbf{A})$ are interpreted is given by the algebra $\mathbb{M}_{n}(\mathbf{A})=\left(M_{n}(\mathbf{A}),+, ;, \mathbf{0}, \mathbf{1}, *\right)$ where $M_{n}(\mathbf{A})$ is the space of $(n \times n)$-matrices over $\mathbf{A}$, the operators + , ; are the usual matrix sum and multiplication, respectively, $\mathbf{0}, \mathbf{1}$ are the zero and the identity matrices, respectively, and $*$ is the operator defined as in $[3,14]$. The matrix representation of a program expresses, for each pair of states $s, s^{\prime}$, the weight (e.g. probability, cost, uncertainty) of the program going from $s$ to $s^{\prime}$.

$\mathcal{G} \mathcal{D} \mathcal{L}(\mathbf{A})$-models for a signature (Prop, $\Pi)$, denoted by $\operatorname{Mod}^{\mathcal{G} \mathcal{L}(\mathbf{A})}(\Pi$, Prop), consists of tuples $\mathcal{A}=\left(W, V,\left(\mathcal{A}_{\pi}\right)_{\pi \in \Pi}\right)$ where $W$ is a finite set (of states), $V:$ Prop $\times W \rightarrow A$ is a valuation function, and $\mathcal{A}_{\pi} \in M_{n}(\mathbf{A})$, with $n$ standing for the cardinality of $W$.

The interpretation of a program $\pi \in \operatorname{Prg}(\Pi)$ in a model $\mathcal{A} \in \operatorname{Mod}^{\mathcal{G D} \mathcal{L}(\mathbf{A})}(\Pi$, Prop) is recursively defined, from the set of atomic programs $\left(\mathcal{A}_{\pi}\right)_{\pi \in \Pi}$, as $\mathcal{A}_{\pi ; \pi^{\prime}}=\mathcal{A}_{\pi} ; \mathcal{A}_{\pi^{\prime}}, \mathcal{A}_{\pi+\pi^{\prime}}=\mathcal{A}_{\pi}+\mathcal{A}_{\pi^{\prime}}$ and $\mathcal{A}_{\pi^{*}}=\mathcal{A}_{\pi}^{*}$.

Satisfaction. The (graded) satisfaction relation, for a model $\mathcal{A} \in \operatorname{Mod}^{\mathcal{G D} \mathcal{L}(\mathbf{A})}(\Pi$, Prop), with A complete, consists of a function $\models: W \times \operatorname{Fm}^{\Gamma(\mathbf{A})}(\Pi$, Prop $) \rightarrow A$ recursively defined as follows:

$-(w \models \top)=\top$

$-(w \models \perp)=\perp$

- $(w \models p)=V(p, w)$, for any $p \in$ Prop

$-\left(w \models \rho \wedge \rho^{\prime}\right)=(w \models \rho) \cdot\left(w \models \rho^{\prime}\right)$

$-\left(w \models \rho \vee \rho^{\prime}\right)=(w \models \rho)+\left(w \models \rho^{\prime}\right)$

- $\left(w \models \rho \rightarrow \rho^{\prime}\right)=(w \models \rho) \rightarrow\left(w \models \rho^{\prime}\right)$

- $\left(w \models \rho \leftrightarrow \rho^{\prime}\right)=\left(w \models \rho \rightarrow \rho^{\prime}\right) ;\left(w \models \rho^{\prime} \rightarrow \rho\right)$

- $(w \models\langle\pi\rangle \rho)=\sum_{w^{\prime} \in W}\left(\mathcal{A}_{\pi}\left(w, w^{\prime}\right) ;\left(w^{\prime} \models \rho\right)\right)$

$-(w \models[\pi] \rho)=\prod_{w^{\prime} \in W}\left(\mathcal{A}_{\pi}\left(w, w^{\prime}\right) \rightarrow\left(w^{\prime} \models \rho\right)\right)$

The (graded) satisfaction in a state gives the degree of certainty of a formula in such a state. For instance $M, w \models\langle\pi\rangle \rho$ is the degree of confidence on the verification of $\rho$ on the execution of $\pi$. It is relevant to note that $\mathcal{G D} \mathcal{L}(\mathbf{A})$ is a generalisation of PDL, for each action lattice $\mathbf{A}$. In particular, by considering the Boolean lattice, the generated logic $\mathcal{G} \mathcal{D} \mathcal{L}(\mathbf{2})$ coincides with PDL. 


\section{Multi-valued concurrent dynamic logic}

Before presenting the construction of the logic, we introduce the mathematical formalism to define the model where programs will be interpreted.

\subsection{Fuzzy binary multirelations}

Definition 3 (Fuzzy set and fuzzy relation [24]). Given a set $X$ and $a$ complete residuated lattice $\mathbf{L}$, a fuzzy subset of $X$ is a function $\phi: X \rightarrow L$. $\phi(x)$, defines the membership degree of $x$ in $\phi$. The set of all fuzzy subsets of $X$ is denote as $L^{X}$. The support of $\phi$ is a fuzzy subset $\psi$ such that $\psi(x)>0$, $\forall x \in X$. Given sets $X, Y$, a fuzzy binary relation over $X, Y$ is a function $\mu: X \times Y \rightarrow L . \mu(x, y)$ is interpreted as the truth value of how elements $x, y$ are related by $\mu$.

Since an action lattice is an extension of a residuated lattice, the concepts of both fuzzy set and fuzzy relation can be defined as well for the former. Such is the case for all the remaining formalisms introduced in this paper.

Definition 4 (Fuzzy binary multirelation). Given a set $X$ and a complete action lattice A over carrier $A$, a fuzzy binary multirelation $R$ over $X$ is a set $R \subseteq X \times A^{X}$. The following operations for fuzzy binary multirelations are defined:

- $R \cup S$ as the union of $R$ and $S$;

- R.S $=\left\{(a, \phi) \mid \phi(c)=\sum_{\left(a, \phi_{a}\right) \in R}\left(\prod_{\left(b, \phi_{b}\right) \in S} \phi_{a}(b) ; \phi_{b}(c)\right)\right\}$

$-R \cap S=\left\{\left(a, \phi_{R} \cup \phi_{S}\right) \mid\left(a, \phi_{R}\right) \in R\right.$ and $\left.\left(a, \phi_{S}\right) \in S\right\}$, where $\phi_{R} \cup \phi_{S}$ is the union of fuzzy sets $\phi_{R}$ and $\phi_{s}$, as defined in [24];

$-R^{*}=\bigcup\left\{R^{n}: n \geq 0\right\}$.

We denote by $M(X)$ the set of all fuzzy binary multirelations over $X$.

Note, particularly, how this definition generalises the concept of binary multirelations, replacing 2 (of Definition 1) by $\mathbf{A}$. This structure supports a set of truth values beyond the Boolean. Therefore, a program is modelled by an execution with multiple "arrows" leaving a state into a set of states in parallel, with a (possible different) fuzziness degree associated with each "arrow". Note that if $\mathbf{A}$ is the Boolean lattice $\mathbf{2}$, any fuzzy binary multirelation $R \subseteq X \times \mathbf{2}^{X}$ is a binary multirelation. Since the goal is still to model programs as binary input-output relations, only the binary case is considered, and thus the remaining of this paper refers to fuzzy binary multirelations simply as fuzzy multirelations. Another aspect that is relevant for the formalisation of the logics is the restriction to fuzzy multirelations $R \subseteq X \times A^{X}$ where the fuzzy set $\phi$ in $A^{X}$ is defined such that $\phi(x)>0, \forall x \in X$.

The operations on fuzzy multirelations somehow mimic the classic case. Clearly operator $\cup$ corresponds to the classical set union. For the sequential composition, the expression for $\phi$ computes the weight of an execution that 
starts in a state $a$, arrives at a set of intermediate states $\phi_{a}$ and ends in a set of states $\varphi_{b}$. The parallel composition considers the union of fuzzy sets for computing the external choice, which is just a generalisation of set union used in CPDL.

\subsection{Parametric construction of multi-valued concurrent dynamic logics}

Each complete action lattice $\mathbf{A}$ induces a multi-valued concurrent propositional dynamic logic $\mathcal{G C D} \mathcal{L}(\mathbf{A})$, with weighted computations interpreted over $\mathbf{A}$. Its signature, formulæ, semantics and satisfaction relation are presented below.

Signatures. Signatures of $\mathcal{G C D} \mathcal{L}(\mathbf{A})$ are pairs $(\Pi$, Prop) corresponding to the denotations of atomic programs and propositions, respectively.

Formulæ. The set of programs, denoted by $\operatorname{Prg}(\Pi)$, consists of all expressions generated by $\pi \ni \pi_{0}|\pi ; \pi| \pi \cap \pi|\pi+\pi| \pi^{*}$, for $\pi_{0} \in \Pi$. Given a signature ( $\Pi$, Prop), the $\mathcal{G C D} \mathcal{L}(\mathbf{A})$-formulæ for ( $\Pi$, Prop), denoted by

$\mathrm{Fm}^{\Gamma(\mathbf{A})}$ ( $\Pi$, Prop), are the ones generated by the grammar $\rho \ni \top|\perp| p|\rho \vee \rho| \rho \wedge \rho|\rho \rightarrow \rho| \rho \leftrightarrow \rho \mid\langle\pi\rangle \rho$, for $p \in$ Prop and $\pi \in \operatorname{Prg}(\Pi)$.

Semantics. The semantic domain is the set of all fuzzy multirelations over a set of states $W$, denoted by $M(W)$, and the operations given in Definition 4 .

$\mathcal{G C D} \mathcal{L}(\mathbf{A})$-models for a signature $(\Pi$, Prop) are tuples $M=(W, V, \llbracket-\rrbracket)$ where $W$ is a set of states, $V$ is a valuation function $V:$ Prop $\times W \rightarrow A$ and $\llbracket-\rrbracket$ attributes a fuzzy multirelation $R \subseteq W \times A^{W}$ to each atomic program.

The interpretation of a program $\pi \in \operatorname{Prg}(\Pi)$ in a model $M$ is recursively defined as:

$\llbracket \pi ; \pi^{\prime} \rrbracket=\llbracket \pi \rrbracket \cdot \llbracket \pi^{\prime} \rrbracket, \llbracket \pi \cap \pi^{\prime} \rrbracket=\llbracket \pi \rrbracket \cap \llbracket \pi^{\prime} \rrbracket, \llbracket \pi+\pi^{\prime} \rrbracket=\llbracket \pi \rrbracket \cup \llbracket \pi^{\prime} \rrbracket$ and $\llbracket \pi^{*} \rrbracket=\llbracket \pi \rrbracket^{*}$.

The satisfaction relation for a model $M=(W, V, \llbracket-\rrbracket)$ is given by the valuation function $\models_{\mathcal{G C D} \mathcal{L}}: W \times \mathrm{Fm}^{\Gamma(\mathbf{A})}(\Pi$, Prop $) \rightarrow A$ recursively defined as:

$$
\begin{aligned}
& \text { - }\left(w \models_{\mathcal{G C D} \mathcal{L}} \top\right)=\perp \\
& -(w \models \mathcal{G C D} \mathcal{L} \perp)=\top \\
& \text { - }(w \models \mathcal{G C D} \mathcal{L} p)=V(p, w) \text {, for any } p \in \text { Prop } \\
& -\left(w \models_{\mathcal{G C D} \mathcal{L}} \rho \wedge \rho^{\prime}\right)=\left(w \models_{\mathcal{G C D} \mathcal{L}} \rho\right) \cdot\left(w \models_{\mathcal{G C D} \mathcal{L}} \rho^{\prime}\right) \\
& \text { - }\left(w \models \mathcal{G C D} \mathcal{L} \rho \vee \rho^{\prime}\right)=(w \models \mathcal{G C D} \mathcal{L} \rho)+\left(w \models \mathcal{G C D} \mathcal{L} \rho^{\prime}\right) \\
& -\left(w \models_{\mathcal{G C D} \mathcal{L}} \rho \rightarrow \rho^{\prime}\right)=\left(w \mid=_{\mathcal{G C D} \mathcal{L}} \rho\right) \rightarrow\left(w \models_{\mathcal{G C D} \mathcal{L}} \rho^{\prime}\right) \\
& \text { - }\left(w \models_{\mathcal{G C D} \mathcal{L}} \rho \leftrightarrow \rho^{\prime}\right)=\left(w=_{\mathcal{G C D} \mathcal{L}} \rho \rightarrow \rho^{\prime}\right) ;\left(w \models_{\mathcal{G C D} \mathcal{L}} \rho^{\prime} \rightarrow \rho\right) \\
& -\left(w \models_{\mathcal{G C D} \mathcal{L}}\langle\pi\rangle \rho\right)=\sum_{\phi \mid(w, \phi) \in \llbracket \pi \rrbracket}\left(\prod _ { u \in U } \left(\phi(u) ;\left(u \models_{\mathcal{G C D} \mathcal{L} \rho)))}\right.\right.\right.
\end{aligned}
$$

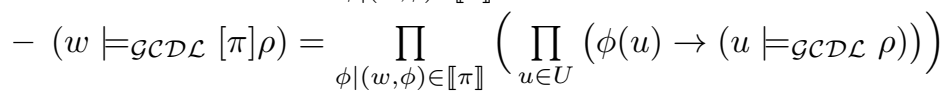

where $U \subseteq W$. We say that $\rho$ is valid when, for any model $M$, and for each state $w \in W,\left(w \models_{\mathcal{G C D} \mathcal{L}} \rho\right)=\top$. 
The (graded) satisfaction of $\left(w \models_{\mathcal{G C D} \mathcal{L}}\langle\pi\rangle \rho\right)$ is given by the weight of some fuzzy set $\phi$ which is related to state $w$ by some fuzzy multirelation, and the weight of $\rho$ for every state in the domain of $\phi$.

As mentioned in Section 2, the axiomatisation of CPDL was presented as that of PDL, except for one axiom restricted to atomic programs, plus an additional axiom for concurrency. Below we study such an axiomatisation in the models of $\mathcal{G C D} \mathcal{L}(\mathbf{A})$. First, Lemma 1 provides some auxiliary properties used to prove next lemma.

Lemma 1. Let $\mathcal{A}$ be a complete $\mathbb{I}$-action lattice. Then

(1.1) $\left(w=_{\mathcal{G C D} \mathcal{L}} \rho \rightarrow \rho^{\prime}\right)=\top$ iff $\left(w=_{\mathcal{G C D} \mathcal{L}} \rho\right) \leq\left(w=_{\mathcal{G C D} \mathcal{L}} \rho^{\prime}\right)$

(1.2) $\left(w=_{\mathcal{G C D} \mathcal{L}} \rho \leftrightarrow \rho^{\prime}\right)=\top$ iff $\left(w \models_{\mathcal{G C D} \mathcal{L}} \rho\right)=\left(w \models_{\mathcal{G C D} \mathcal{L}} \rho^{\prime}\right)$

Proof. Analogous to [15].

Lemma 2. Let $\mathbf{A}$ be a a complete $\mathbb{I}$-action lattice. The following are valid formulce in any $\mathcal{G C D} \mathcal{L}(\mathbf{A})$ :

(2.1) $\left\langle\pi_{0}\right\rangle\left(\rho \vee \rho^{\prime}\right) \leftrightarrow\left\langle\pi_{0}\right\rangle \rho \vee\left\langle\pi_{0}\right\rangle \rho^{\prime}$

(2.2) $\langle\pi\rangle\left(\rho \wedge \rho^{\prime}\right) \rightarrow\langle\pi\rangle \rho \wedge\langle\pi\rangle \rho^{\prime}$

(2.3) $\left\langle\pi+\pi^{\prime}\right\rangle \rho \leftrightarrow\langle\pi\rangle \rho \vee\langle\pi\rangle \rho$

(2.4) $\langle\pi\rangle \perp \leftrightarrow \perp$

(2.5) $\left\langle\pi \cap \pi^{\prime}\right\rangle \rho \leftrightarrow\langle\pi\rangle \rho \wedge\left\langle\pi^{\prime}\right\rangle \rho$

(2.6) $\left[\pi+\pi^{\prime}\right] \rho \leftrightarrow[\pi] \rho \wedge\left[\pi^{\prime}\right] \rho$

(2.7) $[\pi]\left(\rho \wedge \rho^{\prime}\right) \rightarrow[\pi] \rho \wedge[\pi] \rho^{\prime}$

Proof. The proof uses the definition of the satisfaction relation $\models_{\mathcal{G C D} \mathcal{L}}$ and some axioms and properties of action lattices. The technical details are documented in the extended version of this article [8].

\section{Conclusion}

We took, in this paper, the first step in order to develop a rigorous and systematic formalism for the verification of weighted concurrent systems, motivated by the behaviour of programs in the fuzzy computational paradigm. The approach is based on the combination of some ideas from previous research $[16,20,9]$ to characterise both the computational and logical settings on top of which a proper (axiomatic, denotational and operational) semantics for fuzzy programs will be developed, in future work.

There are numerous research lines left open and worth to pursue in the near future. The most obvious one is the study of a proper complete axiomatisation for the generated logics. In particular, the validity of the remaining axioms of CPDL, namely the ones involving operators ; and *, needs to be analysed in the models of the logic. Another prominent path to follow is the study of the relationships between PDL, CPDL and their graded variants. In one direction, 
we propose to investigate whether $\mathrm{CPDL}$ can be obtained from $\mathcal{G C D} \mathcal{L}(\mathbf{A})$ by taking $\mathbf{2}$ as parameter. Another one would be to study if there is a way to obtain $\mathcal{G D} \mathcal{L}(\mathbf{A})$ as special case of $\mathcal{G C D} \mathcal{L}(\mathbf{A})$, such that there is a correspondence between the operations on fuzzy multirelations and operations on matrices. Additionally, relevant results about decidability and complexity of the logics are naturally in our agenda.

Although we based our definition of sequential composition for fuzzy multirelations in Peleg's definition [6], there are other versions of the operator in the literature. One of them corresponds to the definition introduced for giving semantics to Parikh's game logic [19]

$$
R \cdot S=\{(a, A) \mid \exists B .(a, B) \in R \wedge(\forall b \in B .(b, A) \in S)\}
$$

It is clearly stronger than Peleg's, since it requires that every intermediate state $b$ must be related with the arriving set of states $A$. Another one, the Kleisli composition, was later studied in [6], and is motivated by the Kleisli composition of the powerset monad.

Finally, we propose to adapt the models of the generated logics in order to capture the introduction of assignments of variables to values in a given data domain. The goal is to develop (parametric) dynamic logics for the verification of programs written in a fuzzy imperative programming language, such as [23] or $[2]$.

\section{References}

1. Baltag, A., And Smets, S. The dynamic turn in quantum logic. Synthese 186, 3 (2012), 753-773.

2. Cingolani, P., And Alchlá-Fdez, J. jfuzzylogic: a java library to design fuzzy logic controllers according to the standard for fuzzy control programming. Int. J. Comput. Intell. Syst. 6, sup1 (2013), 61-75.

3. Conway, J. Regular Algebra and Finite Machines. Dover Publications, 1971.

4. Den Hartog, J., AND DE Vink, E. P. Verifying probabilistic programs using a Hoare like logic. Int. J. Found. Comput. Sci. 13, 3 (2002), 315-340.

5. Den Hartog, J. I. Probabilistic Extensions of Semantical Models. PhD thesis, Vrije Universiteit, Vrije, 2002.

6. Furusawa, H., Kawahara, Y., Struth, G., and Tsumagari, N. Kleisli, Parikh and Peleg compositions and liftings for multirelations. J. Log. Algebr. Meth. Program. 90 (2017), 84-101.

7. Furusawa, H., And Struth, G. Taming multirelations. ACM Trans. Comput. Log. 17, 4 (2016), 28:1-28:34.

8. Gomes, L. On the construction of multi-valued concurrent dynamic logic. CoRR abs/1911.00462 (2019).

9. Gomes, L., Madeira, A., And Barbosa, L. S. Generalising KAT to verify weighted computations. CoRR abs/1911.01146 (2019).

10. Hoare, C. A. R., Möller, B., Struth, G., and Wehrman, I. Concurrent Kleene algebra. In CONCUR 2009. Proceedings (2009), M. Bravetti and G. Zavattaro, Eds., vol. 5710 of LNCS, Springer, pp. 399-414. 
11. Jipsen, P., And Moshier, M. A. Concurrent Kleene algebra with tests and branching automata. J. Log. Algebr. Meth. Program. 85, 4 (2016), 637-652.

12. Kozen, D. A probabilistic PDL. J. Comput. Syst. Sci. 30, 2 (1985), 162-178.

13. Kozen, D. On action algebras. In Logic and the Flow of Information (Amsterdam, 1993).

14. Kozen, D. A completeness theorem for Kleene algebras and the algebra of regular events. Information and Computation 110, May 1994 (1994), 366-390.

15. Madeira, A., Neves, R., and Martins, M. A. An exercise on the generation of many-valued dynamic logics. JLAMP 1 (2016), 1-29.

16. Madeira, A., Neves, R., Martins, M. A., and Barbosa, L. S. A dynamic logic for every season. In Formal Methods: Foundations and Applications. Proceedings (2014), C. Braga and N. Martí-Oliet, Eds., vol. 8941 of LNCS, Springer, pp. 130145.

17. Martin, C. E., Curtis, S. A., And Rewitzky, I. Modelling angelic and demonic nondeterminism with multirelations. Sci. Comput. Program. 65, 2 (2007), 140-158.

18. McIver, A., Rabehaja, T. M., And Struth, G. An event structure model for probabilistic concurrent kleene algebra. In Logic for Programming, Artificial Intelligence, and Reasoning - 19th International Conference, LPAR-19, Stellenbosch, South Africa, December 14-19, 2013. Proceedings (2013), K. L. McMillan, A. Middeldorp, and A. Voronkov, Eds., vol. 8312 of Lecture Notes in Computer Science, Springer, pp. 653-667.

19. PARIKH, R. Propositional game logic. In 24th Annual Symposium on Foundations of Computer Science (1983), IEEE Computer Society, pp. 195-200.

20. Peleg, D. Concurrent dynamic logic. J. ACM 34, 2 (1987), 450-479.

21. Platzer, A. Logical Analysis of Hybrid Systems - Proving Theorems for Complex Dynamics. Springer, 2010.

22. QIAO, R., Wu, J., WANG, Y., AND GAO, X. Operational semantics of probabilistic Kleene algebra with tests. Proceedings - IEEE Symposium on Computers and Communications (2008), 706-713.

23. Vetterlein, T., Mandl, H., And Adlassnig, K. Fuzzy arden syntax: A fuzzy programming language for medicine. Artificial Intelligence in Medicine 49, 1 (2010), 1-10.

24. ZADEH, L. Fuzzy sets. Information and Control 8, 3 (1965), 338 - 353. 